\title{
Contrasting roles of the RSC and ISWI/CHDI chromatin remodelers in RNA polymerase II elongation and termination
}

\author{
Josefina Ocampo, ${ }^{1,2,3}$ Răzvan V. Chereji, ${ }^{1,2}$ Peter R. Eriksson, ${ }^{1}$ and David J. Clark ${ }^{1}$ \\ ${ }^{1}$ Division of Developmental Biology, Eunice Kennedy Shriver National Institute of Child Health and Human Development, National \\ Institutes of Health, Bethesda, Maryland 20892, USA
}

\begin{abstract}
Most yeast genes have a nucleosome-depleted region (NDR) at the promoter and an array of regularly spaced nucleosomes phased relative to the transcription start site. We have examined the interplay between RSC (a conserved essential SWI/SNFtype complex that determines NDR size) and the ISWI, CHDI, and ISW2 nucleosome spacing enzymes in chromatin organization and transcription, using isogenic strains lacking all combinations of these enzymes. The contributions of these remodelers to chromatin organization are largely combinatorial, distinct, and nonredundant, supporting a model in which the +1 nucleosome is positioned by RSC and then used as a reference nucleosome by the spacing enzymes. Defective chromatin organization correlates with altered RNA polymerase II (Pol II) distribution. RSC-depleted cells exhibit low levels of elongating Pol II and high levels of terminating Pol II, consistent with defects in both termination and initiation, suggesting that RSC facilitates both. Cells lacking both ISWl and CHDl show the opposite Pol II distribution, suggesting elongation and termination defects. These cells have extremely disrupted chromatin, with high levels of closely packed dinucleosomes involving the second (+2) nucleosome. We propose that ISWI and CHDI facilitate Pol II elongation by separating closely packed nucleosomes.
\end{abstract}

[Supplemental material is available for this article.]

Eukaryotic DNA is packaged into the nucleus in the form of chromatin. The basic structural unit of chromatin is the nucleosome, which contains $\sim 147 \mathrm{bp}$ of DNA wrapped almost twice around a central core histone octamer containing two molecules each of histones H2A, H2B, H3, and H4 (Luger et al. 1997). Nucleosomes are compact, stable structures that inhibit DNA-dependent processes such as transcription. Cells possess chromatin remodeling enzymes that manipulate the repressive properties of chromatin to regulate genes. Chromatin remodeling enzymes use the free energy obtained from ATP hydrolysis to assemble, evict, slide or restructure nucleosomes (Clapier and Cairns 2009; Narlikar et al. 2013; Bartholomew 2014; Swygert and Peterson 2014). Mutations in genes encoding subunits of human remodeling complexes are strongly associated with cancer and other diseases (Narlikar et al. 2013).

Genome-wide nucleosome maps for budding yeast reveal that most promoters are located in nucleosome-depleted regions (NDRs) and that nucleosomes are regularly spaced and phased relative to the transcription start site (TSS) (Lee et al. 2004; Yuan et al. 2005; Jiang and Pugh 2009). RSC is an essential SWI/SNF-type complex homologous to human PBAF (Clapier and Cairns 2009). In vitro, RSC can evict, mobilize, or remodel nucleosomes, and create promoter NDRs in reconstituted chromatin (Lorch et al. 2001; Saha et al. 2002; Shukla et al. 2010; Krietenstein et al. 2016). Depletion of an essential RSC subunit results in growth arrest (Treich and Carlson 1997), major changes in gene expression (Ng et al. 2002), and much reduced overall levels of transcription

\footnotetext{
${ }^{2}$ These authors contributed equally to this work.

${ }^{3}$ Present address: Instituto de Investigaciones en Ingeniería Genética y Biología Molecular "Dr. Héctor N. Torres" (INGEBI-CONICET), C1428ADN Buenos Aires, Argentina

Corresponding author: clarkda@mail.nih.gov

Article published online before print. Article, supplemental material, and publication date are at http://www.genome.org/cgi/doi/10.1101/gr.242032.118.
}

by all three RNA polymerases (Parnell et al. 2008). In RSC-depleted cells, nucleosomes shift into the promoter, resulting in narrowing and partial filling of the NDR (Hartley and Madhani 2009; Ganguli et al. 2014; Rawal et al. 2018a). RSC is preferentially associated with the promoter-flanking -1 and +1 nucleosomes and, to a lesser extent, with the +2 and +3 nucleosomes (Yen et al. 2012). RSC is modestly enriched on highly active genes and at tRNA genes $(\mathrm{Ng}$ et al. 2002; Floer et al. 2010; Ganguli et al. 2014; Spain et al. 2014; Rawal et al. 2018a).

The ISW1, CHD1, and ISW2 remodelers space nucleosomes in vitro, such that CHD1 spaces nucleosomes closer together than ISW1, whereas ISW2 spaces them farther apart (Ito et al. 1997; Tsukiyama et al. 1999; Lusser et al. 2005; Stockdale et al. 2006). In vivo, deletion of both ISW1 and CHD1 results in a major disruption of spacing and phasing (Gkikopoulos et al. 2011; Ocampo et al. 2016). We have proposed that the three spacing enzymes compete to set the spacing on individual genes, and the global average spacing reflects the outcome of this competition (Ocampo et al. 2016). In addition to playing a major role in global chromatin organization, ISW1 and CHD1 suppress histone exchange and cryptic initiation (Cheung et al. 2008; Quan and Hartzog 2010; Tirosh et al. 2010; Gkikopoulos et al. 2011; Pointner et al. 2012; Radman-Livaja et al. 2012; Smolle et al. 2012). Single-gene studies show that CHD1 and ISW1 are involved in transcription through chromatin. CHD1 associates with Pol II elongation factors (Simic et al. 2003) and acts as a termination factor, often in concert with ISW1 or ISW2 (Alén et al. 2002). Two different ISW1 complexes (ISW1a and ISW1b) (Vary et al. 2003) coordinate transcription of the MET16 gene, such that ISW1a positions nucleosomes at the promoter to regulate initiation, whereas ISW1b acts in the coding region to facilitate Pol II elongation and termination (Alén et al. 2002; Morillon et al. 2003). The third

This is a work of the US Government. 
spacing enzyme, ISW2, also limits cryptic initiation, but its contribution to chromatin organization is restricted to inactive genes (Goldmark et al. 2000; Kent et al. 2001; Whitehouse et al. 2007; Ocampo et al. 2016).

Much less is known about the interplay between the SWI/ SNF-type complexes and the nucleosome spacing enzymes. ISW1 and RSC cooperate to remodel the PHO5 promoter (Musladin et al. 2014), but they behave antagonistically at other promoters, where ISW1 partly reverses RSC-mediated widening of the NDR (Parnell et al. 2015). Here, we report a systematic study of the roles of RSC and the three nucleosome spacing enzymes in chromatin organization and transcription by analyzing all possible mutant combinations.

\section{Results}

Combinatorial contributions of the RSC, ISW1, CHD1, and ISW2 remodelers to chromatin organization

Previously, we reported the effects on chromatin organization of all combinations of null mutations in ISW1, CHD1, and ISW2, us- ing high resolution nucleosome maps obtained by micrococcal nuclease (MNase) digestion and paired-end sequencing of nucleosomal DNA (MNase-seq; GEO accession number GSE69400) (Ocampo et al. 2016). To investigate the interplay between RSC and the spacing enzymes, we constructed a second set of eight strains to complete the set of 16 isogenic strains corresponding to all possible combinations of RSC depletion with null mutants in the three spacing enzymes. For simplicity, we will refer to Rsc8-depleted cells as $r s c 8$ cells. Nucleosome phasing analysis relative to the +1 nucleosome on all $\sim 5000$ yeast genes was performed on data from all 16 strains (Fig. 1). The use of the +1 nucleosome as a reference point should give more accurate measurements of nucleosome spacing (defined as the average distance between the centers of neighboring nucleosomes) than using the TSS, although the results are only slightly different (Supplemental Fig. S1).

The data we reported previously (Ocampo et al. 2016) are summarized in Figure 1A. In wild-type cells, there is a deep NDR at the promoter with strong nucleosome phasing on the gene. Both chd $1 \Delta$ and isw $1 \Delta$ cells show broader peaks with reduced amplitudes on gene bodies, indicating weaker nucleosome phasing, whereas isw2 cells show no obvious changes in chromatin
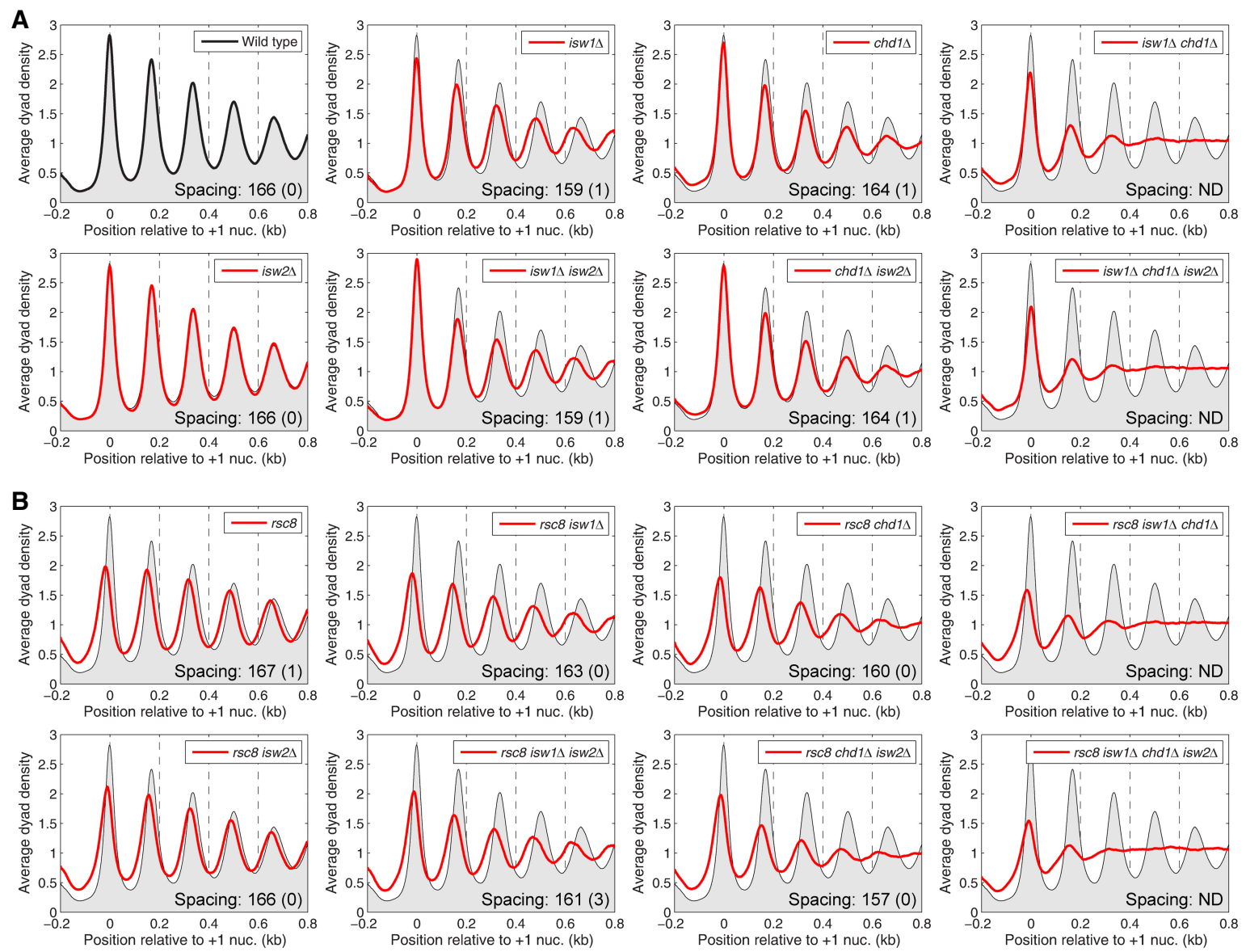

Figure 1. Combinatorial effects of RSC, ISW1, CHD1, and ISW2 remodelers on the chromatin organization of yeast genes. Average nucleosome phasing on all yeast genes relative to the dyad of the +1 nucleosome in wild-type cells. (A) Phasing in wild type, isw $1 \Delta$, chd $1 \Delta$, and isw2 $2 \Delta$ mutants (from Ocampo et al. 2016). (B) Phasing in $r s c 8$ mutants. For comparison, the phasing profile for wild-type cells is shown in all plots (gray shading). Inset values: average spacing determined by regression analysis of the first five peaks, with standard deviation (two biological replicate experiments); (ND) not determined because phasing is poor. (Note: Nucleosome spacing refers to the average distance between the centers of neighboring nucleosomes. An equivalent way of estimating the space between neighboring nucleosomes, or nucleosome repeat length, is by analyzing the periodicity of the bands observed after gel electrophoresis of MNase-digested chromatin.) The sequencing depths of all samples were normalized to one read per bp. See Supplemental Figure S1 for alignment on the TSS.

\section{Genome Research}

www.genome.org 
structure. Nucleosome spacing is reduced from $166 \mathrm{bp}$ in wild-type cells to $159 \mathrm{bp}$ in isw $1 \Delta$ cells: The +1 nucleosome is in the same position as in wild-type cells, but the downstream nucleosomes are shifted toward the promoter. The isw $1 \Delta$ isw $2 \Delta$ and $\operatorname{chd} 1 \Delta$ isw $2 \Delta$ double mutants resemble the isw $1 \Delta$ and chd $1 \Delta$ single mutants, respectively, consistent with a minimal contribution from the isw $2 \Delta$ mutation. The isw $1 \Delta$ chd $1 \Delta$ double mutant shows a major perturbation of nucleosome organization (Gkikopoulos et al. 2011). Both double mutants involving isw $1 \Delta$ show the reduced nucleosome spacing observed in the single mutant, although the effect is not as clear in the isw $1 \Delta$ chd $1 \Delta$ mutant because the phasing is very poor. Finally, the isw $1 \Delta$ chd $1 \Delta$ isw $2 \Delta$ triple mutant is essentially the same as the isw $1 \Delta$ chd $1 \Delta$ double mutant, again consistent with a minimal global contribution from ISW2. Thus, ISW1 and $\mathrm{CHD} 1$ are the major phasing and spacing enzymes. Because the spacing in isw1 $1 \Delta$ cells is short, we inferred previously that CHD1 sets short spacing and that ISW1 makes it longer (Ocampo et al. 2016). We also proposed that ISW1 activity may be partially dependent on CHD1 activity, in order to explain the fact that spacing in chd1 $1 \Delta$ cells is not longer than that of wild type, as predicted by our simple model.

The new data, for the $r s c 8$ strains, are presented in Figure $1 \mathrm{~B}$. In $r s c 8$ cells, as expected from previous studies, all of the nucleosomes in the upstream and downstream arrays shift toward the promoter with consequent narrowing and filling of the NDR, with no change in spacing (Hartley and Madhani 2009; Ganguli et al. 2014; Rawal et al. 2018a). All eight of the $r s c 8$ strains show the same narrowing of the NDR and shift of the +1 nucleosome, indicating that RSC is required for setting the correct positions of the +1 and -1 nucleosomes. Furthermore, the nucleosomal array shifts toward the promoter in all of the $r s c 8$ strains for which spacing can be measured (i.e., all strains except those with both isw1 $1 \Delta$ and $\operatorname{chd} 1 \Delta$, in which phasing is heavily disrupted).

The contributions of the spacing enzymes to chromatin structure in $r s c 8$ strains are also mostly as expected from the single mutants. The $r s c 8$ is $w 1 \Delta$ and the $r s c 8$ chd $1 \Delta$ double mutants both show the weaker phasing typical of the isw $1 \Delta$ and $c h d 1 \Delta$ single mutants, whereas the $r s c 8$ isw $2 \Delta$ double mutant is similar to the $r s c 8$ single mutant, indicating little or no contribution of ISW2. Thus, chromatin organization in the $r s c 8$ double mutants is the combination of the effects observed in the single mutants. This conclusion is supported by analysis of the triple mutants. The $r s c 8$ chd1 1 isw $2 \Delta$ and the $r s c 8$ isw $1 \Delta$ isw $2 \Delta$ triple mutants resemble their respective double mutants without isw $2 \Delta$ (Fig. 1B, cf. upper and lower rows). The $r s c 8 i s w 1 \Delta$ chd $1 \Delta$ triple mutant is essentially a combination of the array shifts typical of $r s c 8$ and the very poor phasing observed when isw1 $1 \Delta$ and chd $1 \Delta$ are combined. Finally, the quadruple mutant ( $r s c 8$ isw1 $1 \Delta$ chd $1 \Delta$ isw $2 \Delta$ ) resembles the $r s c 8$ isw $1 \Delta$ chd $1 \Delta$ triple mutant, as expected given that isw $2 \Delta$ has no discernible effect in the other mutants. In conclusion, to a first approximation, the global chromatin organization of the quadruple mutant is the sum of the separate contributions of the four remodelers.

A quantitative analysis of the average nucleosome spacing reveals that spacing changes in the $r s c 8$ isw $1 \Delta$ and $r s c 8$ chd $1 \Delta$ double mutants do not follow the combinatorial rule, although the deviations are small (Fig. 1). The $r s c 8$ isw $1 \Delta$ double mutant shows a smaller reduction in spacing (163 bp) than expected from the combination of the $r s c 8$ (167 bp; same as wild type) and isw1 $1459 \mathrm{bp}$ ) mutations (Fig. 1). Conversely, the spacing in the $r s c 8$ chd1 $\Delta$ double mutant $(160 \mathrm{bp})$ is significantly less than in the chd1 $1 \Delta$ mutant (164 bp). Thus, $r s c 8$ partially suppresses the effect of $i s w 1 \Delta$ on spac- ing, but enhances the effect of $c h d 1 \Delta$. However, the global average spacing hides the fact that different genes have different spacing (Ocampo et al. 2016). Data for all of the mutants are presented as histograms of the percentage of genes with a given spacing (Fig. 2). In wild-type cells, genes exhibit a broad spacing distribution, ranging from $140 \mathrm{bp}$ to $>200 \mathrm{bp}$, although most genes are in the $155-180 \mathrm{bp}$ range, with a single peak at $\sim 166 \mathrm{bp}$, as expected from the average (Fig. 1). The spacing distribution in $r s c 8$ cells is somewhat narrower than in wild-type cells even though the average spacing is the same as in wild type (Fig. 2). This effect is consistent with reduced levels of transcription in $r s c 8$ cells (Parnell et al. 2008), because very active genes have disrupted chromatin with extremely short or extremely long spacing (for some examples, see Supplemental Fig. S2; Ocampo et al. 2016). Although the narrowing effect is also observed in the $r s c 8$ isw2 $\Delta$ double mutant, it is not observed in any of the other $r s c 8$ mutants, indicating that both ISW1 and CHD1 are important for maintaining the narrower range of nucleosome spacing on individual genes in $r s c 8$ cells.

\section{Loss of remodelers generally increases chromatin disruption}

Nucleosome phasing occurs when regularly spaced nucleosomes are positioned relative to a fixed point in the DNA sequence, such as the TSS. In an array of perfectly positioned nucleosomes on a specific gene, the $+1,+2,+3$ nucleosomes, etc., would adopt exactly the same positions in all cells with a fixed length of linker DNA. In reality, these arrays are far from perfect, as is clear from the width of each nucleosome dyad peak in phasing plots (Fig. 1). We used the width of each peak to quantify the degree of phasing in the mutants, as described previously (Ocampo et al. 2016). The phasing parameter $(\sigma)$ is related to the width of the Gaussian curve fit to each nucleosome peak on the average gene, such that higher values of $\sigma$ indicate weaker phasing ( $\sigma=0$ indicates a perfectly positioned array). Physically, the phasing value reflects both cellto-cell heterogeneity in the +1 nucleosome position (rotational positioning) and in the linker length, which follows the $(10 n+5)$ rule, where $n$ is an integer and predicts linker lengths of $5,15,25$ bp, etc. (Lohr and Van Holde 1979; Brogaard et al. 2012; Chereji et al. 2018). Thus, wild-type and isw $2 \Delta$ cells have $\sigma$ values of $\sim 15$ and $\sim 16$, respectively, whereas $r s c 8$, isw $1 \Delta$, and $\operatorname{chd} 1 \Delta$ have weaker phasing ( 18, $\sim 19$, and $\sim 20$, respectively) and isw $1 \Delta$ chd1 $\Delta$ cells have much worse phasing $(\sigma=\sim 28)$ (Supplemental Fig. S3). The slightly weaker phasing in the $r s c 8$ single mutant reflects the fact that the upstream shift of the nucleosomal array toward the promoter does not occur on all genes (Ganguli et al. 2014). The $r s c 8$ double mutants have worse phasing than the single mutants: $r s c 8$ isw2 $2 \Delta, r s c 8$ isw $1 \Delta$, and $r s c 8$ chd1 $1 \Delta$ have $\sigma=\sim 19, \sim 22$, and $\sim 25$, respectively. In general, the loss of each remodeler results in weaker phasing, indicating increasing chromatin disorganization.

\section{Rsc8-depleted cells exhibit altered gene expression patterns}

Cells lacking RSC activity have much lower transcriptional activity than wild-type cells (Parnell et al. 2008). We compared the Pol II distributions in 10 of our 16 strains by ChIP-seq for the Rpb3 subunit (Supplemental Fig. S4A). The Pol II occupancy patterns of the isw $1 \Delta$, chd1 $\Delta$, and isw2 $\Delta$ single mutants are very similar to wild type (Spearman's rank correlation $\sim 0.9$ ); very few genes show changes greater than twofold relative to wild type, although smaller differences could be physiologically important. Even the isw1 $\Delta$ chd1 $1 \Delta$ double mutant, which shows very poor global phasing, is similar to wild type $(R>0.9)$, as shown previously (Gkikopoulos 
A

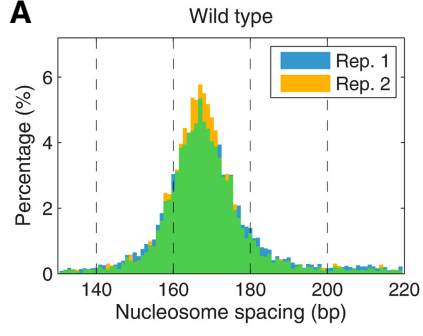

isw2 $\Delta$

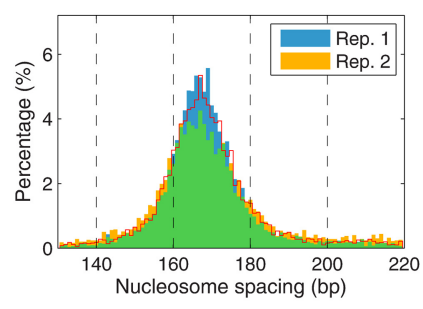

B

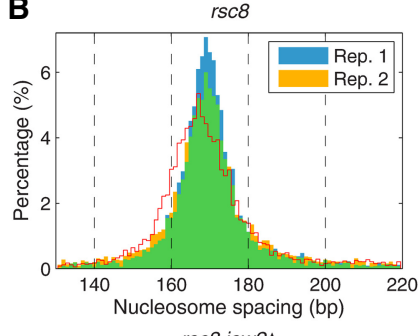

rsc8 isw2 $\Delta$

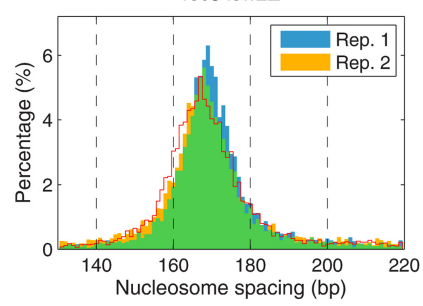

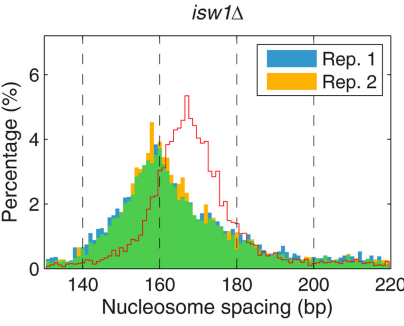

isw14 isw2 $\Delta$
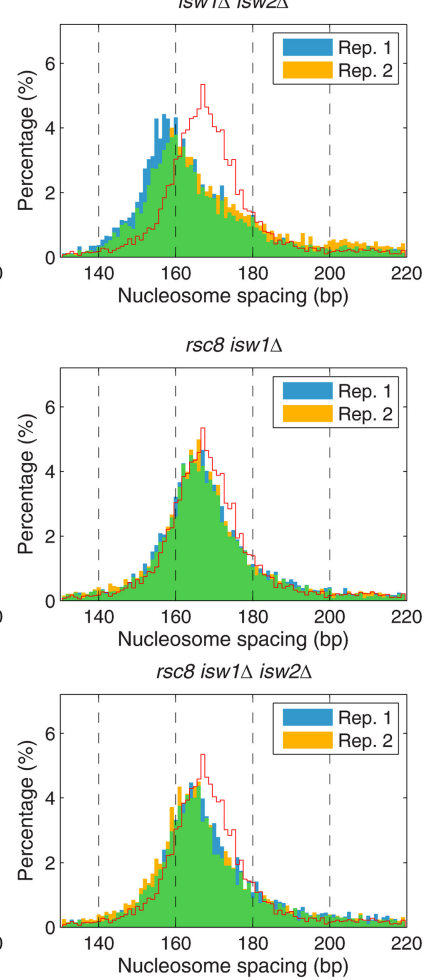
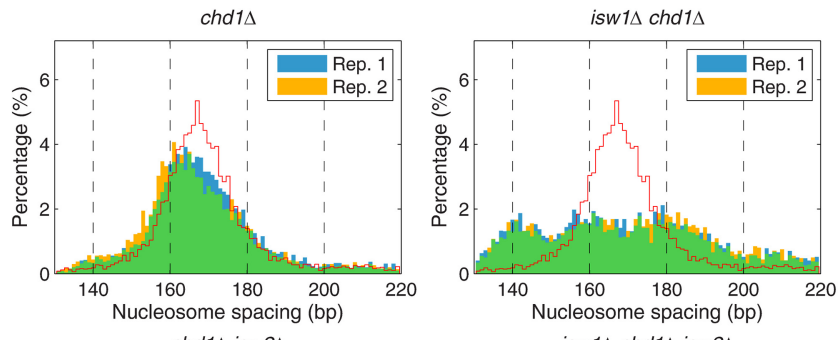

chd1 1 isw2 $2 \Delta$
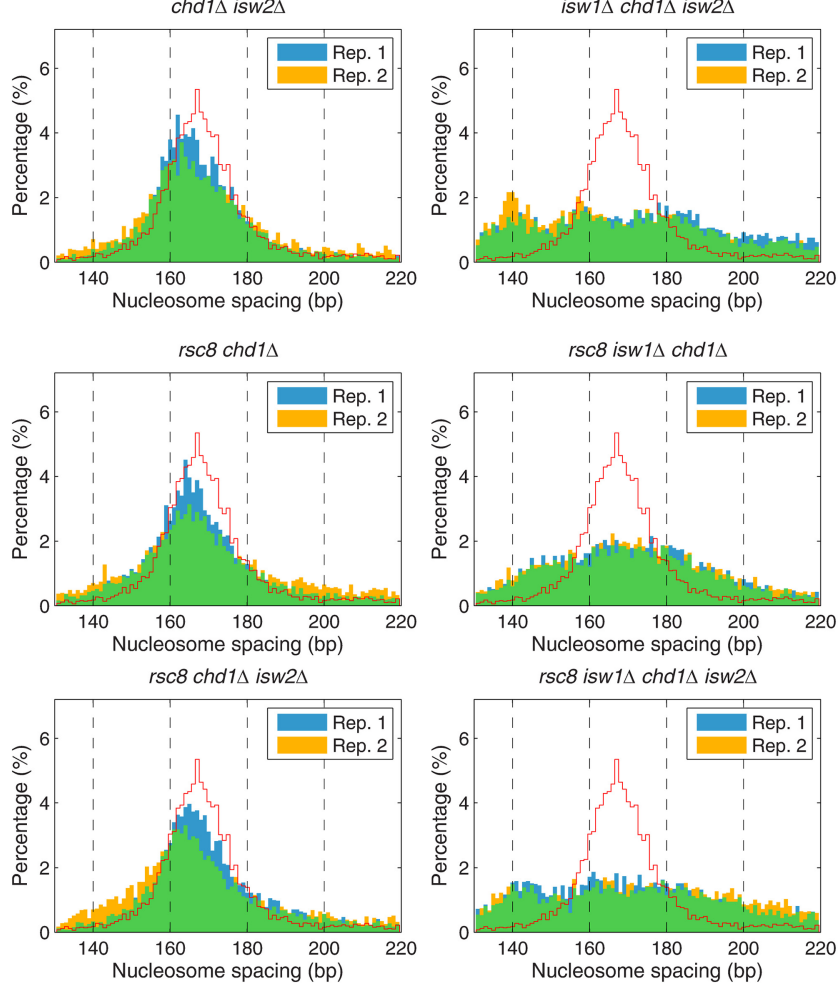

Figure 2. Nucleosome spacing distributions in the chromatin remodeler mutants. Histograms of the percentage of genes having a given average spacing (1-bp bins). Data from two biological replicates are shown, indicated by blue and yellow bars; overlap is indicated by green bars. For comparison, the wildtype histogram is indicated by the red line. (A) Spacing distributions in wild type, isw $1 \Delta$, chd1 $\Delta$, and isw2 $2 \Delta$ mutants (from Ocampo et al. 2016 ). (B) Spacing distributions in $r s c 8$ mutants.

et al. 2011; Ocampo et al. 2016). The gene expression pattern in $r s c 8$ cells is less similar to that of wild type and the other single mutants $(R<0.8)$ (Supplemental Fig. S4A). Hierarchical clustering analysis shows that the gene expression patterns of all three $r s c 8$ double mutants are relatively poorly correlated with wild type but correlate well with the $\operatorname{rsc} 8$ single mutant; the $R$ values appear to define two sets of closely correlated strains $(R>$ 0.9 ): those like wild type and those like $r s c 8$ strains (Supplemental Fig. S4A).

Because RSC-depleted cells arrest in the G2/M phase of the cell cycle (Tsuchiya et al. 1992; Treich and Carlson 1997), we considered the possibility that the changes in gene expression in $r s c 8$ cells are primarily due to growth arrest. However, we found that nocodazole-induced arrest of wild-type cells in G2/M has only minor effects on gene expression ( $R \sim 0.9$ compared with no nocodazole) (Supplemental Fig. S4B). In contrast, the gene expression patterns of cells arrested in G2/M by nocodazole or by Rsc8 depletion are quite different $(R \sim 0.75)$ (Supplemental Fig. S4B), indicating that Rsc8 depletion substantially alters gene expression patterns independently of cell cycle arrest.

\section{RSC and ISW1/CHDI have opposite effects on the levels of elongating and terminating Pol II}

It is surprising that genes in $r s c 8$ cells are associated with significant amounts of Pol II because transcriptional activity, as measured by RNA levels in RSC-depleted cells, is low (Parnell et al. 2008). We hypothesized that Pol II might be paused in $r s c 8$ cells, such that it is engaged but not actively transcribing. Promoter-proximal pausing of Pol II is common in higher eukaryotes, but it is not generally observed in yeast (Mayer et al. 2017). In wild-type cells, Pol II levels are low at the promoter and high on the gene (elongating Pol II), with a well-resolved dip at the transcript termination site (TTS), and a peak just downstream from the TTS that sometimes trails into the next gene, corresponding to terminating Pol II (Cole et al. 2014; Rawal et al. 2018b). Pol II transcribes through the TTS, which refers to the mRNA cleavage site, where poly(A) is added and the mRNA is released. Pol II is still engaged downstream from the TTS and continues to transcribe before it is forced to dissociate from the DNA by Pol II termination factors (Porrua and Libri 2015).

\section{Genome Research}

www.genome.org 
To determine whether the genic distribution of Pol II is altered in the mutants, we divided genes into divergent, tandem, and convergent pairs to resolve promoters from termination sites. We constructed heat maps of Pol II occupancy for divergent gene pairs aligned on the TSS and sorted by the distance between their TSSs (Fig. 3A; Supplemental Fig. S5). Divergent gene pairs in wild type and in all of the mutants have relatively low levels of Pol II at promoters and higher levels on the gene, with little evidence for promoter-proximal pausing. Tandem gene pairs were sorted by the distance between the TTS of the upstream gene and the TSS of the downstream gene (Fig. 3A). In $r s c 8$ cells, a peak of Pol II is apparent in the termination regions of the upstream genes of tandem pairs, sometimes reaching as far as the TSS of the downstream gene (Fig. 3A, upper rows in the lower panel). A weak peak corresponding to terminating Pol II is apparent in wild-type cells but almost absent in the isw $1 \Delta$ chd $1 \Delta$ mutant (Fig. 3A). Thus, Pol II accumulates at the $3^{\prime}$ ends of genes in $r s c 8$ cells, but its levels are relatively low at $3^{\prime}$ ends in isw $1 \Delta$ chd $1 \Delta$ cells, suggesting that these mutants have opposite termination defects.

We confirmed these observations by examining tandem and convergent gene pairs (Fig. 3B,C; Supplemental Fig. S6). The average Pol II distributions on tandem gene pairs relative to the TTS of the upstream gene show that the terminating Pol II peak is more prominent relative to the level of elongating Pol II in $r s c 8$ cells than in wild-type cells and less prominent in isw $1 \Delta$ chd $1 \Delta$ cells (Fig. 3B). The level of terminating Pol II in the chd1 $\Delta$ single mutant is slightly lower than in wild type, suggesting that CHD1 contributes more to the larger effect observed in the isw1 $1 \Delta$ chd1s double mutant than ISW1 (Supplemental Fig. S6A). Convergent gene pairs exhibit two poorly separated peaks flanking the TTS in all strains; these peaks represent terminating Pol II on both genes in the pair, such that the upstream peak belongs to the downstream gene and vice versa (Fig. 3B,C; Supplemental Fig. S6).
A
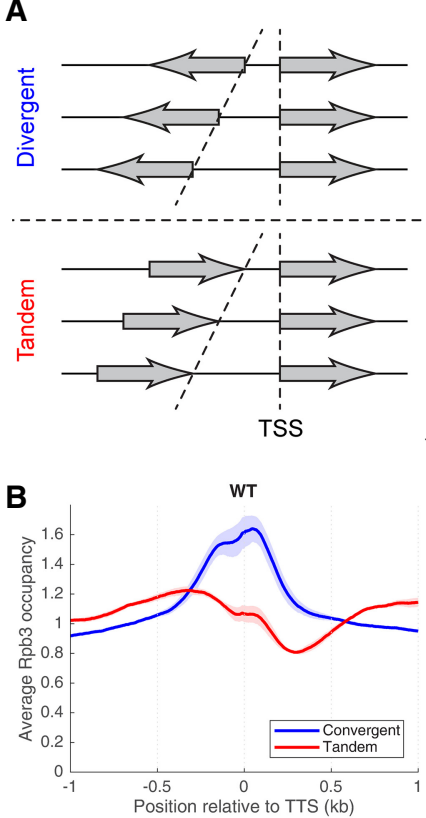

C
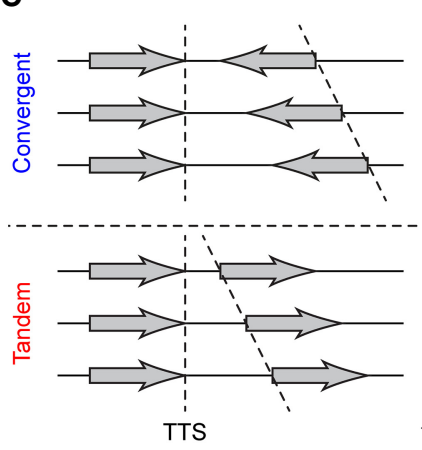
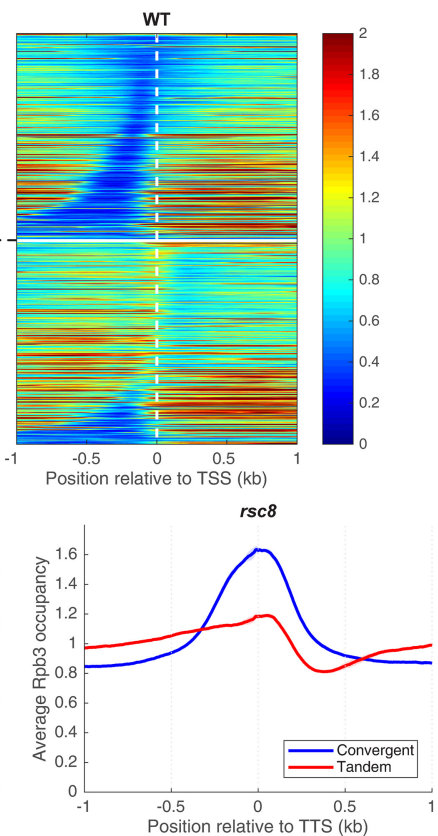

WT

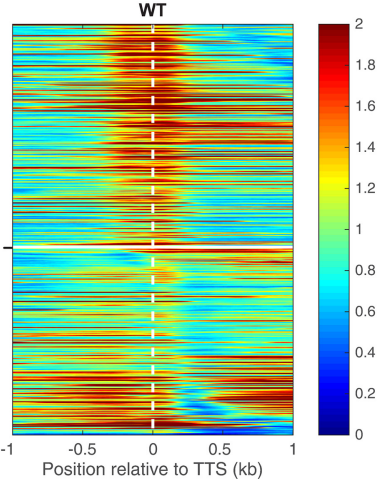

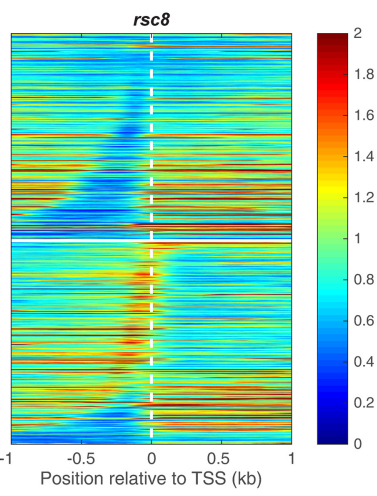

isw1 $1 \Delta$ chd1 $\triangle$

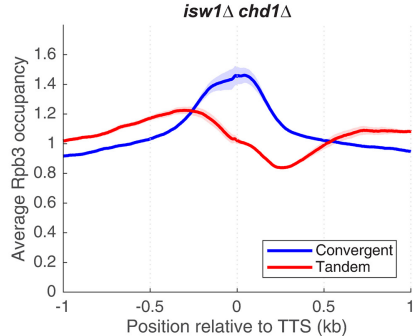

$r s c 8$

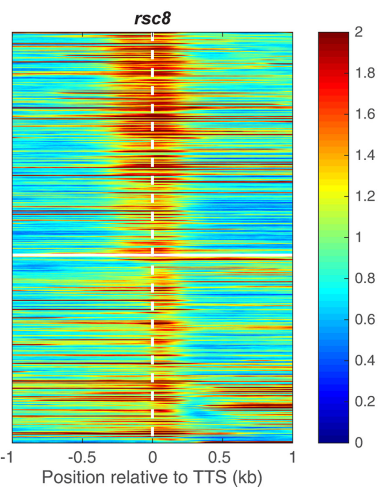

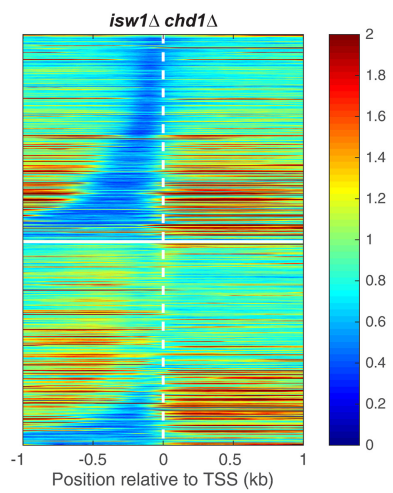

2h Nocodazole (Treated)

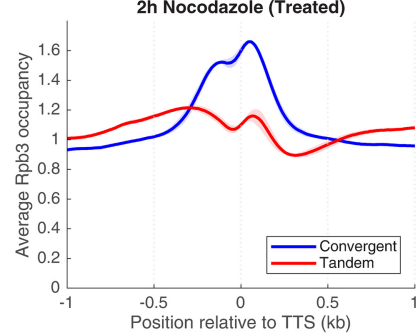

isw1 $\Delta$ chd1 $\Delta$

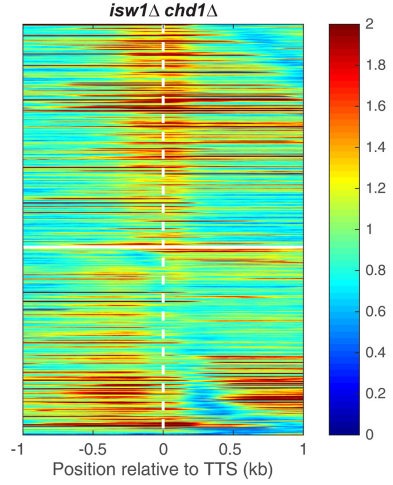

Figure 3. RSC and ISW1/CHD1 have opposite effects on the levels of elongating and terminating Pol II. ( $A$ ) Pol II occupancy heat maps. Genes were divided into divergent (top) and tandem (bottom) pairs (separated by the white horizontal line), which were then sorted by intergenic distance and aligned on the TSS of the downstream gene (dashed vertical line). Data for wild type, rsc8, and isw1 chd1 double mutant cells. Rpb3 ChIP-seq data for DNA fragments of $300 \mathrm{bp}$ or less normalized to the genomic average (set at 1 ). Shaded areas indicate the range of biological replicate data. (B) Aggregate plots for Pol II occupancy (IP/Input) relative to the TTS of the upstream gene for convergent and tandem gene pairs in wild type, $r s c 8$, isw $1 \Delta$ chd $1 \Delta$ double mutant, and nocodazole-treated wild-type cells. (C) Heat maps for the data in $B$, aligned on the TTS of the upstream gene and sorted by the distance to the TSS of the downstream gene. The white horizontal line separates convergent (top) from tandem gene pairs (bottom). Equivalent plots for the other strains are shown in Supplemental Figures S5, S6. 
The accumulation of Pol II downstream from the TTS in $r s c 8$ cells suggests that termination is inhibited (i.e., that RSC facilitates Pol II termination), and the low levels of mRNA in RSC-depleted cells (Parnell et al. 2008) may at least partly reflect a termination defect. This Pol II termination defect cannot be attributed to the arrest of these cells in $\mathrm{G} 2 / \mathrm{M}$, because nocodazole-induced arrest of wild-type cells at the same stage of the cell cycle has little effect on the genic distribution of Pol II (Fig. 3B; Supplemental Fig. S6).

For the isw $1 \Delta$ chd $1 \Delta$ double mutant, the simplest interpretation is that Pol II dissociation is facilitated, implying that ISW1 and CHD1 normally inhibit termination and/or dissociation. It is unlikely that Pol II transcribes farther downstream from the TTS before dissociating, because a lower, extended peak is expected but not observed (Fig. 3). Alternatively, Pol II elongation may be inhibited in isw1 $1 \Delta$ chd $1 \Delta$ cells, resulting in higher levels of Pol II on the gene than downstream from the TTS (see below). The effect of RSC on termination is epistatic to the separate effects of ISW1 and CHD1, because the $r s c 8$ isw $1 \Delta$ and $r s c 8$ chd $1 \Delta$ double mutants and the quadruple mutant all have higher levels of terminating Pol II than the isw1 $1 \Delta$ chd $1 \Delta$ double mutant (Supplemental Figs. S5, S6).

\section{Chromatin organization around the TTS in rsc8 and iswls chd1s cells}

We examined nucleosome occupancy at the TTS to determine whether altered chromatin structure can account for the termination defect in $r s c 8$ cells (Fig. 4; Supplemental Fig. S7). In wild-type cells, there is an NDR at the TTS between tandem genes, which is reproducibly shallower in $r s c 8$ and isw $1 \Delta$ chd $1 \Delta$ cells (Fig. 4A; Supplemental Fig. S7). However, the depth of the $3^{\prime}$ NDR is strongly dependent on the extent of MNase digestion, because 3' UTRs tend to be AT-rich and therefore nucleosomes covering 3' UTRs are digested faster than nucleosomes with normal AT-content (Schwabish and Struhl 2004; Chereji et al. 2017). Consequently, it is difficult to rule out the possibility that these differences in 3 -NDR depth are due to small differences in the extent of MNase digestion. In any case, higher nucleosome occupancy is observed at the TTSs of convergent genes in both $r s c 8$ and isw $1 \Delta$ chd $1 \Delta$ cells (Fig. 4), even though these mutants have quite different levels of terminating Pol II, suggesting that higher nucleosome occupancy does not necessarily inhibit termination.

\section{High levels of closely packed dinucleosomes involving the +2 nucleosome are present in iswl $\Delta$ chdls cells}

There is an apparent reduction in nucleosome occupancy on the downstream gene of tandem gene pairs in wild-type cells (Fig. 4A). This is not actually the case, because the NDR at the promoter belonging to the downstream gene is located at a variable distance from the TTS, resulting in reduced average occupancy (see heat maps, Fig. 4B). However, this effect is extreme for tandem genes

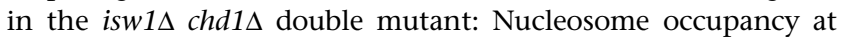
the $5^{\prime}$ ends of downstream genes is much reduced (Fig. 4A), with a disproportionately greater effect on the +2 nucleosome that is
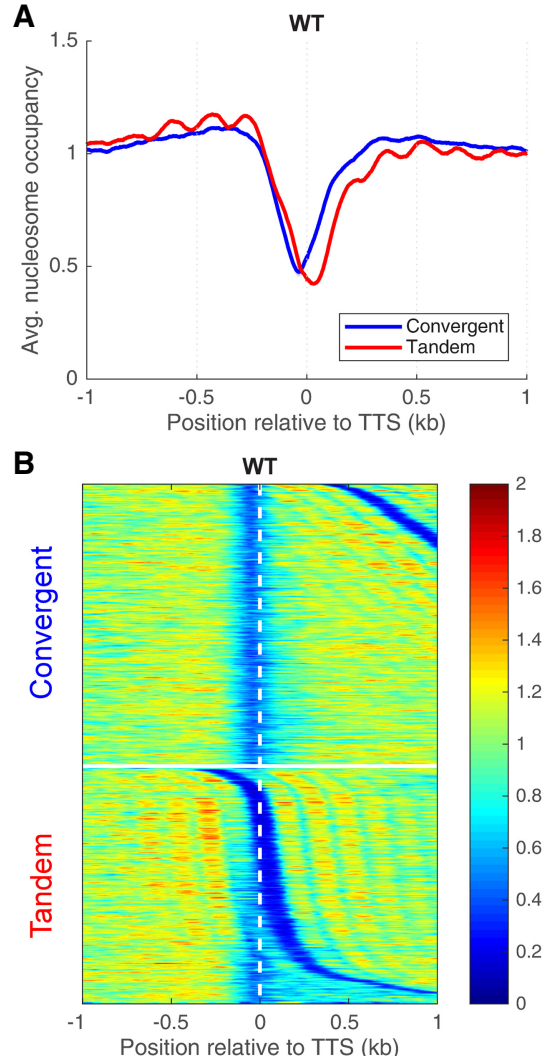
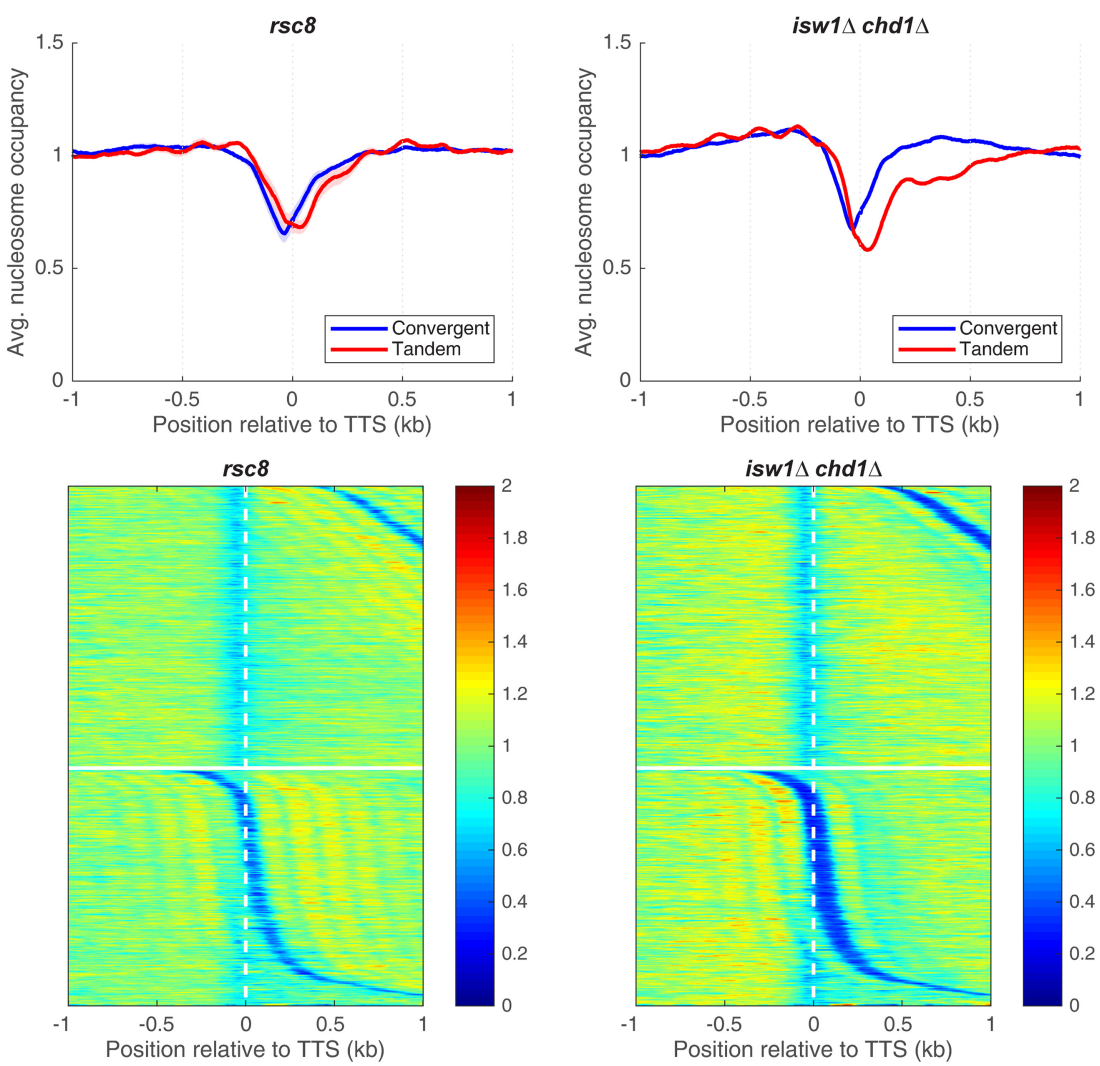

Figure 4. Chromatin organization in the vicinity of the TTS in $r s c 8$ and isw $1 \Delta$ chd $1 \Delta$ cells. $(A)$ Nucleosome occupancy aggregate plots for convergent and tandem gene pairs aligned on the TTS of the upstream gene. MNase-seq data (for fragments of 120-160 bp) normalized to the genomic average (set at 1). $(B)$ Heat maps for the data in $A$, aligned on the TTS of the upstream gene and sorted by the distance to the TSS of the downstream gene. The white horizontal line separates convergent from tandem gene pairs. Equivalent plots for the other strains are shown in Supplemental Figure S7.

\section{Genome Research}

www.genome.org 
obvious in the heat maps (Fig. 4B). This observation suggests that nucleosomes are missing from the $5^{\prime}$ ends of genes in the isw $1 \Delta$ chd $1 \Delta$ double mutant. However, an alternative explanation for the reduced nucleosome occupancy is suggested by the close proximity of two nucleosomes at the 5' end of MET16 in an isw1s mutant (Morillon et al. 2003), which prevents MNase from cutting between them. We considered the possibility that nucleosome occupancy is normal in the isw $1 \Delta$ chd $1 \Delta$ double mutant, and that nucleosomes are sometimes pushed together, eliminating the linker DNA. This model predicts that the missing nucleosomes are actually present as MNaseresistant dinucleosomes. Such dinucleosomes would have been eliminated from our data when the mononucleosome band was excised from the gel.

To test the model, we sequenced MNase-digested DNA that had not been gel-purified. Wild-type mononucleosomes show a normal phasing pattern relative to the +1 nucleosome, whereas mononucleosomes from the isw $1 \Delta$ chd $1 \Delta$ double mutant show weak phasing and reduced occupancy of the +1 nucleosome and, in particular, of the +2 nucleosome (Fig. 5A), as observed for gelpurified mononucleosomes (as expected from Fig. 4). Wild-type dinucleosomes show a fairly even occupancy similar to that of mononucleosomes, except for the +1 nucleosome, which is reduced, probably because the probability of MNase cutting upstream in the NDR is always high, increasing the probability of releasing the +1 nucleosome as a mononucleosome (Fig. 5A). This could account for the slightly higher occupancy of the +1 nucleosome in the mononucleosome fraction (Fig. 5A). In contrast, the isw $1 \Delta$ chd $1 \Delta$ double mutant shows a strong enrichment of dinucleosomes that include the +2 nucleosome (i.e., the $+1 /+2$ and $+2 /+3$ dinucleosomes) (Fig. 5A). We separated divergent and tandem gene pairs in heat maps aligned on the +1 nucleosome dyad and sorted by intergenic distance (Fig. $5 B)$. In wild-type cells, phasing with respect to the NDR is obvious for both mononucleosomes and dinucleosomes. In the isw1 $\Delta$ chd1 $1 \Delta$ double mutant, the +2 nucleosome is clearly depleted from the mononucleosome fraction and enhanced in the dinucleosomes. In the tandem gene heat maps, nucleosome phasing at the $3^{\prime}$ end of the upstream gene is only mildly affected in the isw $1 \Delta$ chd $1 \Delta$ double mutant, even though phasing on the downstream gene is heavily disrupted. In conclusion, the +2 nucleosome is often located immediately adjacent to the +1 or the +3 nucleosome in isw1 $1 \Delta$ chd1 $\Delta$ cells. Thus, ISW1 and CHD1 together prevent close packing of the +2 nucleosome by maintaining regular spacing.
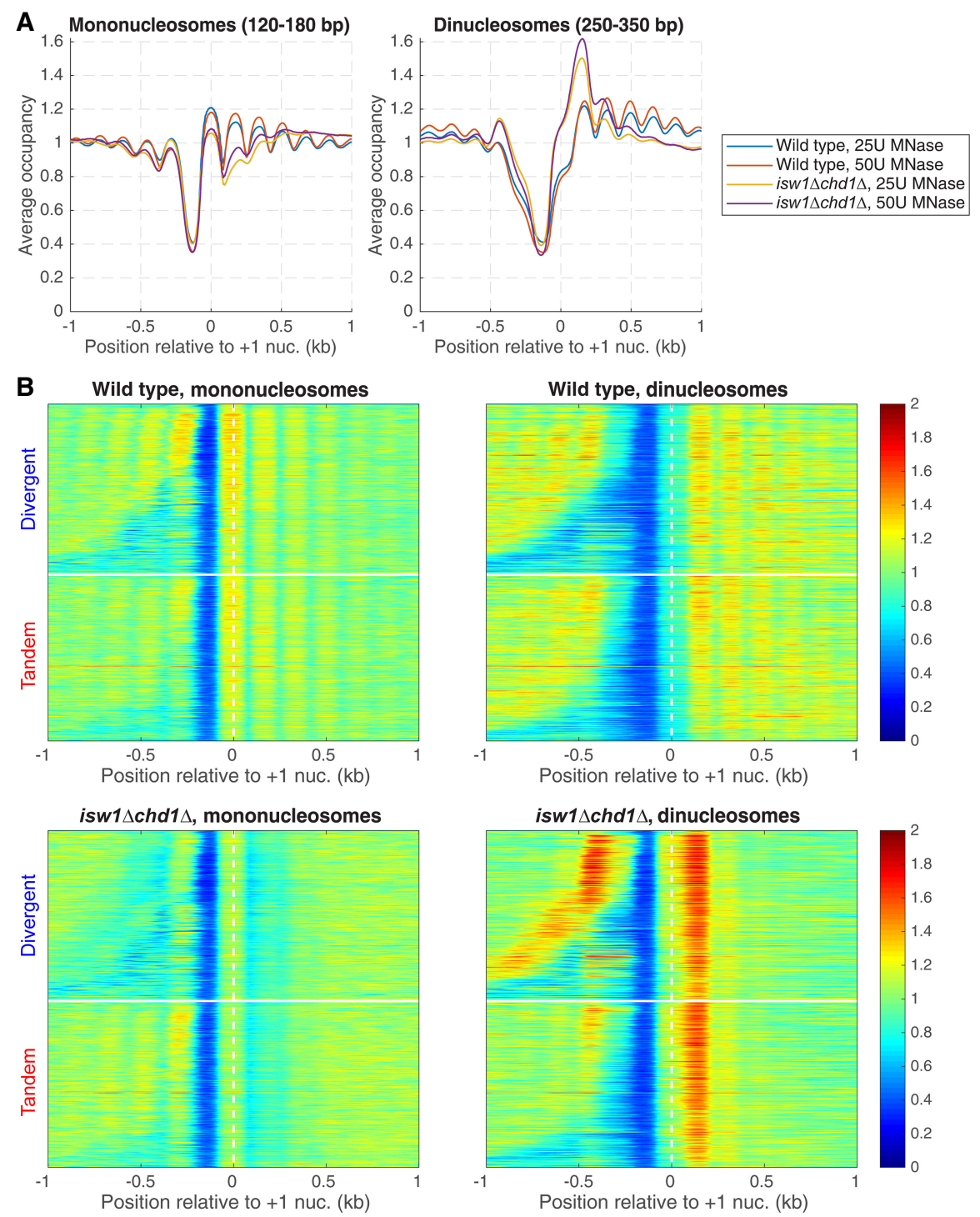

Figure 5. Closely packed dinucleosomes at the $5^{\prime}$ ends of genes in isw $1 \Delta$ chd $1 \Delta$ cells. MNase-seq data for wild-type and isw $1 \Delta$ chd1 $1 \Delta$ cells obtained without gel-purification of mononucleosomal DNA (two levels of digestion: 25 and 50 units MNase). (A) Nucleosome occupancy aggregate plots for mononuNormalized to the genomic average (set at 1). (B) Heat maps for the data in $A$, separated into divergent and tandem gene pairs, sorted by intergenic distance and aligned on the +1 nucleosome of the downstream gene. The white horizontal line separates divergent from tandem gene pairs.

\section{Discussion}

Combinatorial contributions to chromatin organization by RSC, ISWI, CHDI, and ISW2

We report a systematic analysis of chromatin organization and gene expression in isogenic strains representing all possible combinations of RSC depletion with null mutations in three nucleosome spacing enzymes. We confirm that, in RSC-depleted cells, the upstream and downstream nucleosomal arrays shift toward the promoter with narrowing and filling of the NDR (Hartley and Madhani 2009; Ganguli et al. 2014). These effects persist when RSC depletion is combined with any of the spacing enzyme null mutations. RSC-depleted cells also have a narrower nucleosome 
spacing distribution, indicating that there are fewer genes with extreme spacing. Because extreme spacing correlates with heavy transcription (Ocampo et al. 2016), we attribute the narrower spacing distribution in RSC-depleted cells to reduced transcription (Parnell et al. 2008). Generally speaking, the chromatin organization in the double, triple, and quadruple mutants can be predicted by combining the effects of the single mutations. Thus, RSC depletion results in nucleosome array shifts into the promoter, loss of ISW1 or CHD1 results in weaker phasing, whereas loss of ISW2 has only minor global effects. The exception to this rule concerns nucleosome spacing, since the global average spacings are not as predicted from a simple combination of the single mutants. That is, all isw1 $1 \Delta$ mutants should have short spacing and all chd1 $1 \Delta$ mutants should have longer spacing than wild type. Our previous proposal that ISW1 activity may depend partly on CHD1 activity may explain this effect (Ocampo et al. 2016). The INO80 remodeling complex also contributes to spacing, since ino80 $\Delta$ cells have shorter spacing than wild type (Udugama et al. 2011; Yen et al. 2012; van Bakel et al. 2013; Krietenstein et al. 2016).

In summary, we envisage that RSC determines the width of the NDR, either directly by setting the positions of the -1 and +1 nucleosomes (Krietenstein et al. 2016), or indirectly by determining the size of the barrier complex (Mavrich et al. 2008; Jiang and Pugh 2009; Chereji et al. 2017). Then ISW1, CHD1, and, to a much lesser extent, ISW2, compete to space nucleosomes on the gene (Ocampo et al. 2016) using the +1 nucleosome as a reference position. The outcome of the competition on each gene determines its spacing and depends on its transcriptional activity, since CHD1 (the short-spacing enzyme) is associated with active transcription (Simic et al. 2003) and ISW2 (the long-spacing enzyme) affects only relatively inactive genes (Ocampo et al. 2016).

\section{RSC and gene expression}

The gene expression pattern in RSC-depleted cells is quite different from that of wild-type cells, which may reflect both direct and indirect effects on chromatin organization. Indirect effects may be expected as a result of growth arrest, which occurs primarily in the G2/M phase of the cell cycle (Tsuchiya et al. 1992; Treich and Carlson 1997). However, our nocodazole experiments indicate that cell cycle arrest in G2/M has only minor effects on transcription, unlike RSC depletion, which also arrests cells at the spindle assembly checkpoint (Tsuchiya et al. 1992). We note that growth arrest as a result of Rsc8 depletion may also affect nucleosome positioning. Direct effects of RSC are more difficult to predict because RSC localization data are somewhat contradictory. The major contribution of RSC to chromatin organization is at the NDR, as discussed above, predicting that RSC binds at or near promoters in vivo. MNase-ChIP-seq experiments are consistent with this expectation: RSC is bound to the promoter-flanking +1 and -1 nucleosomes, although it is also enriched at the +2 and +3 nucleosomes (Yen et al. 2012). On the other hand, conventional ChIP-seq experiments indicate that RSC is not generally enriched at promoters, but modestly enriched on active genes ( $\mathrm{Ng}$ et al. 2002; Floer et al. 2010; Ganguli et al. 2014; Spain et al. 2014; Rawal et al. 2018a), suggesting that RSC affects elongation rather than initiation. RSC may affect both stages of transcription by adjusting the distance between the +1 and -1 nucleosomes and evicting nucleosomes from NDRs (Rawal et al. 2018a) to facilitate transcription complex formation and by moving or removing nucleosomes on active genes to regulate elongation.

\section{RSC stimulates Pol II termination/dissociation}

We find that RSC has an unexpected stimulatory effect on Pol II termination and/or dissociation after transcription. Dissociation of Pol II appears to be slower in $r s c 8$ cells than in wild-type cells, since ChIP-seq experiments measure bound Pol II. However, the actual defect could be slow termination of transcription rather than slow dissociation. Slow dissociation seems less likely than a termination defect, because the "closed" Pol II complex is much less stable than the transcribing "open" complex. Inhibition of termination alone cannot account for the observed Pol II distribution in RSC-depleted cells, because a pileup of elongating polymerases behind the terminating polymerase would be expected if dissociation is prevented, leading to a higher level of elongating polymerase, which is not observed. A plausible scenario is that transcript initiation is also inhibited in RSC-depleted cells, perhaps due to partial disassembly of the barrier complex (Ganguli et al. 2014; Chereji et al. 2017) and defects in nucleosome eviction or sliding at promoters (Rawal et al. 2018a). An initiation defect is consistent with the fact that RSC is not enriched on terminal nucleosomes (Yen et al. 2012). We speculate that, in wild-type cells, Pol II termination or dissociation might be slow in order to facilitate reinitiation by Pol II (i.e., transfer of Pol II from just downstream from the TTS back to the promoter without release into the nucleoplasm) (Shandilya and Roberts 2012; Cole et al. 2014). Such a mechanism has been proposed to explain fast reinitiation by Pol III (Arimbasseri et al. 2013). In this speculative model, loss of RSC activity would result in the formation of a defective transcription complex in the NDR, reducing initiation and increasing the residence time of Pol II just downstream from the TTS as it waits for transfer back to the promoter.

\section{ISW1 and CHD1 resolve closely packed dinucleosomes}

A role for all three nucleosome spacing enzymes in Pol II termination is suggested by single-gene studies (Alén et al. 2002; Morillon et al. 2003). Transcription run-on assays indicate that Pol II fails to terminate correctly in chd1, isw 1 , and isw 2 mutants, although the strength of the effect depends on the gene. Instead, Pol II continues transcription into the downstream promoter, interfering with its expression (Alén et al. 2002). Pol II ChIP data for a reporter gene (Morillon et al. 2003) indicate that, unlike in wild-type cells, there is a very strong Pol II peak at the $3^{\prime}$ end in isw 1 cells, suggesting a termination defect. However, our data for the average gene in isw1 $\Delta$ cells show the opposite effect: a decrease in Pol II just downstream from the TTS, not the large increase observed by Morillon et al. (2003), although the small number of genes examined by Morillon et al. (2003) may be atypical.

We show here that chromatin disruption in isw $1 \Delta$ chd $1 \Delta$ cells involves the close packing of nucleosomes on genes, particularly of the +2 nucleosome, such that it is positioned immediately adjacent to the +1 nucleosome or to the +3 nucleosome. This observation implies that an important function of ISW1 and CHD1 is to prevent close packing by spacing the nucleosomes out. An interesting question is whether these closely packed dinucleosomes are formed by spontaneous sliding or by other remodelers. Support for the latter model comes from observations that, in vitro, human SWI/SNF can generate "altosomes" (Ulyanova and Schnitzler 2005), and yeast RSC can form "overlapping dinucleosomes" lacking one H2A-H2B dimer (Engeholm et al. 2009; Kato et al. 2017). If RSC forms the dinucleosomes we have observed, we would expect chromatin organization in the $r s c 8$ isw $1 \Delta$ chd $1 \Delta$ triple mutant to be closer to wild type than to the isw $1 \Delta$ chd $1 \Delta$ double mutant, but this

\section{Genome Research}

www.genome.org 
is not the case, suggesting that RSC is not important for dinucleosome formation, although it may be redundant with SWI/SNF.

Although our data are consistent with a termination defect in isw $1 \Delta$ chd $1 \Delta$ cells, it is unclear how closely packed dinucleosomes near the $5^{\prime}$ end of a gene could facilitate Pol II termination or dissociation at the $3^{\prime}$ end. Instead, we propose that Pol II elongation through closely packed nucleosomes at the $5^{\prime}$ end of the gene is slow, eventually resulting in normal termination (Fig. 6). Other important factors to consider are the high levels of histone exchange and cryptic initiation in the isw $1 \Delta$ chd1 $1 \Delta$ double mutant (Smolle et al. 2012). Close packing of nucleosomes may be linked to histone exchange if one of the nucleosomes loses an H2A-H2B dimer, resulting in octasome-hexasome dimers (Engeholm et al. 2009; Kato et al. 2017). Cryptic antisense transcription may result in collisions with elongating sense polymerases, such that fewer polymerases reach the TTS, which would also increase the amount of Pol II on the gene relative to the region just downstream from the TTS (Fig. 6). Thus, ISW1 and CHD1 maintain a chromatin structure conducive to efficient elongation and termination by resolving dinucleosomes and creating regularly spaced nucleosomal arrays.

\section{Methods}

\section{Yeast strains}

All yeast strains used in this work are listed in Supplemental Table S1. In our previous study of RSC (Ganguli et al. 2014), we used a strain with a GAL promoter driving the essential RSC8 gene on a plasmid (Treich and Carlson 1997), such that the cells grow in galactose medium but eventually arrest when switched to glucose. However, this strain derives from S288C, whereas our isw1 $\Delta$, isw $2 \Delta$, and $c h d 1 \Delta$ mutants are all derived from W303. To maintain isogenicity, we constructed a new GAL-RSC8 strain in the same W303 background. A GAL-RSC8 cassette with three N-terminal HA epitope tags was integrated into the genome instead of using a plasmid. We did not observe any differences in chromatin organization between the two GAL-RSC8 strains. GAL-RSC8 strains were grown in synthetic complete (SC) medium containing $2 \%$ galactose and switched to glucose as described (Ganguli et al. 2014); cells were harvested when growth reached a plateau (after $\sim 7.5 \mathrm{~h}$ in glucose). Rsc8 depletion was verified by immunoblotting using anti-HA-peroxidase antibody clone 3F10 (Roche 12013819001) (Supplemental Fig. S8).
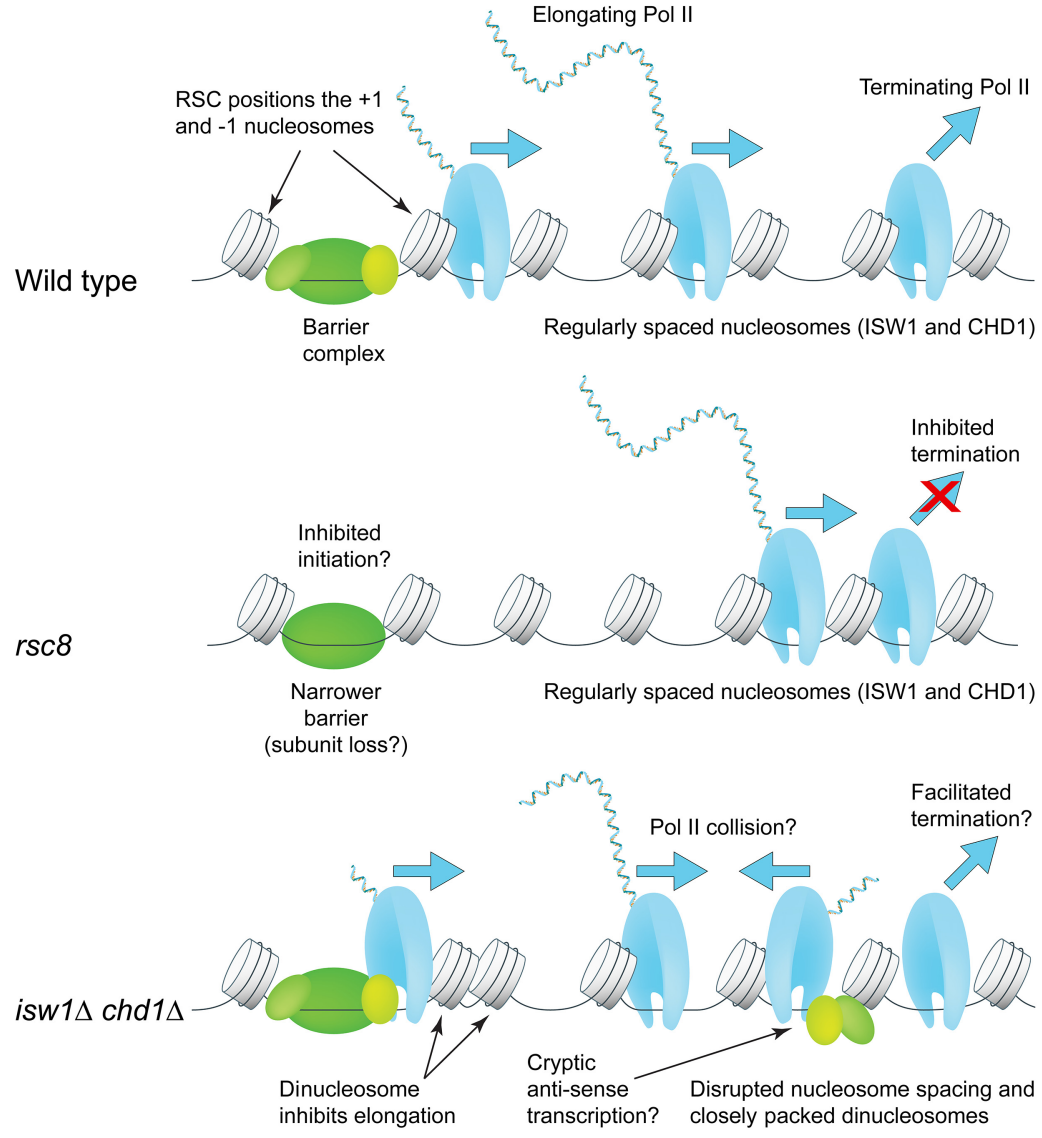

Figure 6. Roles of the RSC, ISW1, and CHD1 remodelers in Pol II initiation, elongation, and termination. RSC widens the promoter NDR by positioning the +1 and -1 nucleosomes farther apart to accommodate a complete barrier complex. CHD1 (short spacing) and ISW1 (longer spacing) compete to set nucleosome spacing on the gene using the +1 nucleosome as a reference. In $r s c 8$ cells, nucleosomes shift toward the promoter but the spacing is the same as wild type; Pol II remains $3^{\prime}$ of the TTS. In isw $1 \Delta$ chd $1 \Delta$ cells, nucleosome spacing is disrupted, with the +2 nucleosome in particular being pushed against the +1 or +3 nucleosome. High cryptic initiation in these cells may result in collisions between transcribing cryptic antisense Pol II and promoter-initiated Pol II (elongation defect). Termination and/or dissociation in these cells may be faster.

\section{MNase-seq and Pol II ChIP-seq}

Nucleosomal DNA was prepared by MNase digestion of isolated nuclei and subjected to paired-end sequencing (Cole et al. 2011, 2012). These experiments, including biological replicates, were performed as described (Ocampo et al. 2016). For dinucleosome analysis, paired-end libraries were prepared from MNase-digested samples without gel-purification of the DNA (both mono- and dinucleosomes were sequenced).

\section{Nocodazole arrest}

Wild-type strain YDC111 (Kim et al. 2006) was grown in SC medium to $\mathrm{A}_{600} \sim 0.25$ and nocodazole (Abcam ab120630) was added to $6 \mu \mathrm{g} / \mathrm{mL}$. Cells were monitored by microscopy for $2 \mathrm{~h}$, by which time the large majority displayed the dumbbell shape characteristic of G2/M arrest. Arrest was confirmed using fluorescence microscopy after staining for DNA using propidium iodide. Cells were fixed with formaldehyde and processed for Pol II ChIP-seq as described (Ocampo et al. 2016).

\section{Bioinformatic analysis}

For phasing analysis, nucleosome sequences in the range of $120-160 \mathrm{bp}$ were used. To facilitate comparisons between different samples, we normalized the sequencing depths of all samples to one read per bp. Details and scripts for estimation of the phasing parameter and nucleosome spacing on individual genes described by Ocampo et al. (2016) and in Supplemental Methods are available as Supplemental Code and at 
https://github.com/rchereji/Nucleosome_spacing_estimation/tree/ GenomeRes_2019.

\section{Data access}

The sequencing data from this study have been submitted to the NCBI Gene Expression Omnibus (GEO; http://www.ncbi .nlm.nih.gov/geo/) under accession numbers GSE73428 and GSE117514.

\section{Acknowledgments}

We thank Alan Hinnebusch for comments on the manuscript. This research was supported by the Intramural Research Program of the NIH (NICHD). We thank the NHLBI Core Facility (Yan Luo, Poching Liu, and Jun Zhu) for paired-end sequencing. This study utilized the high performance computational capabilities of the Biowulf Linux cluster at the National Institutes of Health.

\section{References}

Alén C, Kent NA, Jones HS, O'Sullivan J, Aranda A, Proudfoot NJ. 2002. A role for chromatin remodeling in transcriptional termination by RNA polymerase II. Mol Cell 10: 1441-1452. doi:10.1016/S1097-2765(02) 00778-5

Arimbasseri AG, Rijal K, Maraia RJ. 2013. Transcription termination by the eukaryotic RNA polymerase III. Biochim Biophys Acta 1829: 318-330. doi:10.1016/j.bbagrm.2012.10.006

Bartholomew B. 2014. Regulating the chromatin landscape: structural and mechanistic perspectives. Annu Rev Biochem 83: 671-696. doi:10. 1146/annurev-biochem-051810-093157

Brogaard K, Xi L, Wang JP, Widom J. 2012. A map of nucleosome positions in yeast at base-pair resolution. Nature 486: 496-501. doi:10.1038/ nature 11142

Chereji RV, Ocampo J, Clark DJ. 2017. MNase-sensitive complexes in yeast: nucleosomes and non-histone barriers. Mol Cell 65: 565-577.e3. doi:10. 1016/j.molcel.2016.12.009

Chereji RV, Ramachandran S, Bryson TD, Henikoff S. 2018. Precise genomewide mapping of single nucleosomes and linkers in vivo. Genome Biol 19: 19. doi:10.1186/s13059-018-1398-0

Cheung V, Chua G, Batada NN, Landry CR, Michnick SW, Hughes TR, Winston F. 2008. Chromatin- and transcription-related factors repress transcription from within coding regions throughout the Saccharomyces cerevisiae genome. PLoS Biol 6: e277. doi:10.1371/journal.pbio.0060277

Clapier CR, Cairns BR. 2009. The biology of chromatin remodeling complexes. Annu Rev Biochem 78: 273-304. doi:10.1146/annurev. biochem.77.062706.153223

Cole HA, Howard BH, Clark DJ. 2011. Activation-induced disruption of nucleosome position clusters on the coding regions of Gcn4-dependent genes extends into neighbouring genes. Nucleic Acids Res 39: 95219535. doi:10.1093/nar/gkr643

Cole HA, Howard BH, Clark DJ. 2012. Genome-wide mapping of nucleosomes in yeast using paired-end sequencing. Meth Enzymol 513: 145168. doi:10.1016/B978-0-12-391938-0.00006-9

Cole HA, Ocampo J, Iben JR, Chereji RV, Clark DJ. 2014. Heavy transcription of yeast genes correlates with differential loss of histone H2B relative to $\mathrm{H} 4$ and queued RNA polymerases. Nucleic Acids Res 42: 12512-12522. doi:10.1093/nar/gku1013

Engeholm M, de Jager M, Flaus A, Brenk R, van Noort J, Owen-Hughes T. 2009. Nucleosomes can invade DNA territories occupied by their neighbors. Nat Struct Mol Biol 16: 151-158. doi:10.1038/nsmb.1551

Floer M, Wang X, Prabhu V, Berrozpe G, Narayan S, Spagna D, Alvarez D, Kendall J, Krasnitz A, Stepansky A, et al. 2010. A RSC/nucleosome complex determines chromatin architecture and facilitates activator binding. Cell 141: 407-418. doi:10.1016/j.cell.2010.03.048

Ganguli D, Chereji RV, Iben JR, Cole HA, Clark DJ. 2014. RSC-dependent constructive and destructive interference between opposing arrays of phased nucleosomes in yeast. Genome Res 24: 1637-1649. doi:10. 1101/gr.177014.114

Gkikopoulos T, Schofield P, Singh V, Pinskaya M, Mellor J, Smolle M Workman JL, Barton GJ, Owen-Hughes T. 2011. A role for Snf2-related nucleosome-spacing enzymes in genome-wide nucleosome organization. Science 333: 1758-1760. doi:10.1126/science.1206097

Goldmark JP, Fazzio TG, Estep PW, Church GM, Tsukiyama T. 2000. The Isw2 chromatin remodeling complex represses early meiotic genes upon recruitment by Ume6p. Cell 103: 423-433. doi:10.1016/S00928674(00)00134-3

Hartley PD, Madhani HD. 2009. Mechanisms that specify promoter nucleosome location and identity. Cell 137: 445-458. doi:10.1016/j.cell. 2009.02.043

Ito T, Bulger M, Pazin MJ, Kobayashi R, Kadonaga JT. 1997. ACF, an ISWIcontaining and ATP-utilizing chromatin assembly and remodeling factor. Cell 90: 145-155. doi:10.1016/S0092-8674(00)80321-9

Jiang C, Pugh BF. 2009. Nucleosome positioning and gene regulation: advances through genomics. Nat Rev Genet 10: 161-172. doi:10.1038/ nrg2522

Kato D, Osakabe A, Arimura Y, Mizukami Y, Horikoshi N, Saikusa K, Akashi S, Nishimura Y, Park SY, Nogami J, et al. 2017. Crystal structure of the overlapping dinucleosome composed of hexasome and octasome. Science 356: 205-208. doi:10.1126/science.aak9867

Kent NA, Karabetsou N, Politis PK, Mellor J. 2001. In vivo chromatin remodeling by yeast ISWI homologs Isw1p and Isw2p. Genes Dev 15: 619-626. doi:10.1101/gad.190301

Kim Y, McLaughlin N, Lindstrom K, Tsukiyama T, Clark DJ. 2006. Activation of Saccharomyces cerevisiae HIS3 results in Gcn4p-dependent SWI/SNF-dependent mobilization of nucleosomes over the entire gene. Mol Cell Biol 26: 8607-8622. doi:10.1128/MCB.00678-06

Krietenstein N, Wal M, Watanabe S, Park B, Peterson CL, Pugh BF, Korber P. 2016. Genomic nucleosome organization reconstituted with pure proteins. Cell 167: 709-721.e12. doi:10.1016/j.cell.2016.09.045

Lee CK, Shibata Y, Rao B, Strahl BD, Lieb JD. 2004. Evidence for nucleosome depletion at active regulatory regions genome-wide. Nat Genet 36: 900905. doi:10.1038/ng1400

Lohr D, Van Holde KE. 1979. Organization of spacer DNA in chromatin. Proc Natl Acad Sci 76: 6326-6330. doi:10.1073/pnas.76.12.6326

Lorch Y, Zhang M, Kornberg RD. 2001. RSC unravels the nucleosome. Mol Cell 7: 89-95. doi:10.1016/S1097-2765(01)00157-5

Luger K, Mäder AW, Richmond RK, Sargent DF, Richmond TJ. 1997. Crystal structure of the nucleosome core particle at $2.8 \AA$ resolution. Nature 389: 251-260. doi:10.1038/38444

Lusser A, Urwin DL, Kadonaga JT. 2005. Distinct activities of CHD1 and ACF in ATP-dependent chromatin assembly. Nat Struct Mol Biol 12: 160-166. doi: $10.1038 / \mathrm{nsmb} 884$

Mavrich TN, Ioshikhes IP, Venters BJ, Jiang C, Tomsho LP, Qi J, Schuster SC, Albert I, Pugh BF. 2008. A barrier nucleosome model for statistical positioning of nucleosomes throughout the yeast genome. Genome Res 18: 1073-1083. doi:10.1101/gr.078261.108

Mayer A, Landry HM, Churchman LS. 2017. Pause \& go: from the discovery of RNA polymerase pausing to its functional implications. Curr Opin Cell Biol 46: 72-80. doi:10.1016/j.ceb.2017.03.002

Morillon A, Karabetsou N, O'Sullivan J, Kent N, Proudfoot N, Mellor J. 2003. Isw1 chromatin remodeling ATPase coordinates transcription elongation and termination by RNA polymerase II. Cell 115: 425-435. doi:10.1016/S0092-8674(03)00880-8

Musladin S, Krietenstein N, Korber P, Barbaric S. 2014. The RSC chromatin remodeling complex has a crucial role in the complete remodeler set for yeast PHO5 promoter opening. Nucleic Acids Res 42: 4270-4282. doi:10. 1093/nar/gkt1395

Narlikar GJ, Sundaramoorthy R, Owen-Hughes T. 2013. Mechanisms and functions of ATP-dependent chromatin-remodeling enzymes. Cell 154: 490-503. doi:10.1016/i.cell.2013.07.011

$\mathrm{Ng} \mathrm{HH}$, Robert F, Young RA, Struhl K. 2002. Genome-wide location and regulated recruitment of the RSC nucleosome-remodeling complex. Genes Dev 16: 806-819. doi:10.1101/gad.978902

Ocampo J, Chereji RV, Eriksson PR, Clark DJ. 2016. The ISW1 and CHD1 ATP-dependent chromatin remodelers compete to set nucleosome spacing in vivo. Nucleic Acids Res 44: 4625-4635. doi:10.1093/nar/gkw068

Parnell TJ, Huff JT, Cairns BR. 2008. RSC regulates nucleosome positioning at Pol II genes and density at Pol III genes. EMBO J 27: 100-110. doi:10. 1038/sj.emboj.7601946

Parnell TJ, Schlichter A, Wilson BG, Cairns BR. 2015. The chromatin remodelers RSC and ISW1 display functional and chromatin-based promoter antagonism. eLife 4: e06073. doi:10.7554/eLife.06073

Pointner J, Persson J, Prasad P, Norman-Axelsson U, Strålfors A Khorosjutina O, Krietenstein N, Svensson JP, Ekwall K, Korber P. 2012. CHD1 remodelers regulate nucleosome spacing in vitro and align nucleosomal arrays over gene coding regions in S. pombe. EMBO J 31: 43884403. doi:10.1038/emboj.2012.289

Porrua O, Libri D. 2015. Transcription termination and the control of the transcriptome: why, where and how to stop. Nat Rev Mol Cell Biol 16: 190-202. doi:10.1038/nrm3943

Quan TK, Hartzog GA. 2010. Histone H3K4 and K36 methylation, Chd1 and Rpd3S oppose the functions of Saccharomyces cerevisiae Spt4-Spt5 in transcription. Genetics 184: 321-334. doi:10.1534/genetics.109.111526 Radman-Livaja M, Quan TK, Valenzuela L, Armstrong JA, van Welsem T, Kim T, Lee LJ, Buratowski S, van Leeuwen F, Rando OJ, et al. 2012. A

\section{Genome Research}

www.genome.org 
key role for Chd1 in histone $\mathrm{H} 3$ dynamics at the $3^{\prime}$ ends of long genes in yeast. PLoS Genet 8: e1002811. doi:10.1371/journal.pgen.1002811

Rawal Y, Chereji RV, Qiu H, Ananthakrishnan S, Govind CK, Clark DJ, Hinnebusch AG. 2018a. SWI/SNF and RSC cooperate to reposition and evict promoter nucleosomes at highly expressed genes in yeast. Genes Dev 32: 695-710. doi:10.1101/gad.312850.118

Rawal Y, Chereji RV, Valabhoju V, Qiu H, Ocampo J, Clark DJ, Hinnebusch AG. 2018b. Gcn4 binding in coding regions can activate internal and canonical 5' promoters in yeast. Mol Cell 70: 297-311.e4. doi:10. 1016/j.molcel.2018.03.007

Saha A, Wittmeyer J, Cairns BR. 2002. Chromatin remodeling by RSC involves ATP-dependent DNA translocation. Genes Dev 16: 2120-2134. doi:10.1101/gad.995002

Schwabish MA, Struhl K. 2004. Evidence for eviction and rapid deposition of histones upon transcriptional elongation by RNA polymerase II. Mol Cell Biol 24: 10111-10117. doi:10.1128/MCB.24.23.10111-10117. 2004

Shandilya J, Roberts SGE. 2012. The transcription cycle in eukaryotes: from productive initiation to RNA polymerase II recycling. Biochim Biophys Acta 1819: 391-400. doi:10.1016/j.bbagrm.2012.01.010

Shukla MS, Syed SH, Montel F, Faivre-Moskalenko C, Bednar J, Travers A, Angelov D, Dimitrov S. 2010. Remosomes: RSC generated non-mobilized particles with approximately $180 \mathrm{bp}$ DNA loosely associated with the histone octamer. Proc Natl Acad Sci 107: 1936-1941. doi:10.1073/ pnas.0904497107

Simic R, Lindstrom DL, Tran HG, Roinick KL, Costa PJ, Johnson AD, Hartzog GA, Arndt KM. 2003. Chromatin remodeling protein Chd1 interacts with transcription elongation factors and localizes to transcribed genes. ЕМВО J 22: 1846-1856. doi:10.1093/emboj/cdg179

Smolle M, Venkatesh S, Gogol MM, Li H, Zhang Y, Florens L, Washburn MP, Workman JL. 2012. Chromatin remodelers Isw1 and Chd1 maintain chromatin structure during transcription by preventing histone exchange. Nat Struct Mol Biol 19: 884-892. doi:10.1038/nsmb.2312

Spain MM, Ansari SA, Pathak R, Palumbo MJ, Morse RH, Govind CK. 2014. The RSC complex localizes to coding sequences to regulate Pol II and histone occupancy. Mol Cell 56: 653-666. doi:10.1016/j.molcel.2014. 10.002

Stockdale C, Flaus A, Ferreira H, Owen-Hughes T. 2006. Analysis of nucleosome repositioning by yeast ISWI and Chd1 chromatin remodeling complexes. I Biol Chem 281: 16279-16288. doi:10.1074/jbc. M600682200

Swygert SG, Peterson CL. 2014. Chromatin dynamics: interplay between remodeling enzymes and histone modifications. Biochim Biophys Acta 1839: $728-736$. doi:10.1016/j.bbagrm.2014.02.013
Tirosh I, Sigal N, Barkai N. 2010. Widespread remodeling of mid-coding sequence nucleosomes by Isw1. Genome Biol 11: R49. doi:10.1186/ gb-2010-11-5-r49

Treich I, Carlson M. 1997. Interaction of a Swi3 homolog with Sth1 provides evidence for a Swi/Snf-related complex with an essential function in Saccharomyces cerevisiae. Mol Cell Biol 17: 1768-1775. doi:10.1128/ MCB.17.4.1768

Tsuchiya E, Uno M, Kiguchi A, Masuoka K, Kanemori Y, Okabe S, Mikayawa T. 1992. The Saccharomyces cerevisiae NPS1 gene, a novel CDC gene which encodes a $160 \mathrm{kDa}$ nuclear protein involved in $\mathrm{G}_{2}$ phase control. EMBO J 11: 4017-4026. doi:10.1002/j.1460-2075.1992.tb05495.x

Tsukiyama T, Palmer J, Landel CC, Shiloach J, Wu C. 1999. Characterization of the imitation switch subfamily of ATP-dependent chromatin-remodeling factors in Saccharomyces cerevisiae. Genes Dev 13: 686-697. doi:10. 1101/gad.13.6.686

Udugama M, Sabri A, Bartholomew B. 2011. The INO80 ATP-dependent chromatin remodeling complex is a nucleosome spacing factor. Mol Cell Biol 31: 662-673. doi:10.1128/MCB.01035-10

Ulyanova NP, Schnitzler GR. 2005. Human SWI/SNF generates abundant, structurally altered dinucleosomes on polynucleosomal templates. Mol Cell Biol 25: 11156-11170. doi:10.1128/MCB.25.24.11156-11170.2005

van Bakel H, Tsui K, Gebbia M, Mnaimneh S, Hughes TR, Nislow C. 2013. A compendium of nucleosome and transcript profiles reveals determinants of chromatin architecture and transcription. PLoS Genet 9: e1003479. doi:10.1371/journal.pgen.1003479

Vary JC, Gangaraju VK, Qin J, Landel CC, Kooperberg C, Bartholomew B, Tsukiyama T. 2003. Yeast Isw1p forms two separable complexes in vivo. Mol Cell Biol 23: 80-91. doi:10.1128/MCB.23.1.80-91.2003

Whitehouse I, Rando OJ, Delrow J, Tsukiyama T. 2007. Chromatin remodelling at promoters suppresses antisense transcription. Nature 450: 1031-1035. doi:10.1038/nature06391

Yen K, Vinayachandran V, Batta K, Koerber RT, Pugh BF. 2012. Genomewide nucleosome specificity and directionality of chromatin remodelers. Cell 149: 1461-1473. doi:10.1016/j.cell.2012.04.036

Yuan GC, Liu YJ, Dion MF, Slack MD, Wu LF, Altschuler SJ, Rando OJ. 2005. Genome-scale identification of nucleosome positions in S. cerevisiae. Science 309: 626-630. doi:10.1126/science.1112178

Received July 23, 2018; accepted in revised form January 22, 2019. 


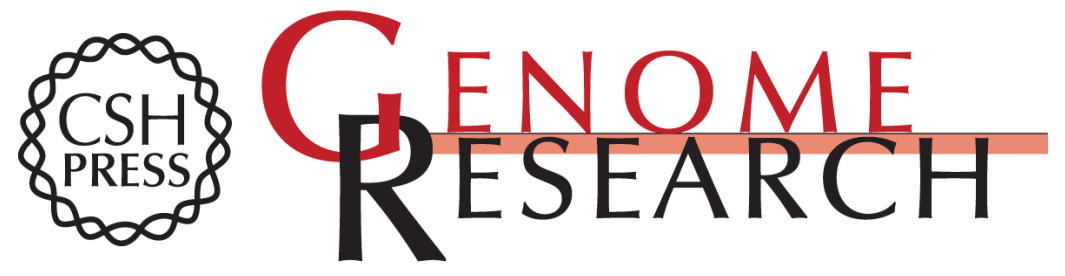

\section{Contrasting roles of the RSC and ISW1/CHD1 chromatin remodelers in RNA polymerase II elongation and termination}

Josefina Ocampo, Razvan V. Chereji, Peter R. Eriksson, et al.

Genome Res. 2019 29: 407-417 originally published online January 25, 2019

Access the most recent version at doi:10.1101/gr.242032.118

Supplemental Material

References

License

Email Alerting Service
http://genome.cshlp.org/content/suppl/2019/02/08/gr.242032.118.DC1

This article cites 63 articles, 22 of which can be accessed free at: http://genome.cshlp.org/content/29/3/407.full.html\#ref-list-1

This is a work of the US Government.

Receive free email alerts when new articles cite this article - sign up in the box at the top right corner of the article or click here.

\section{Affordable, Accurate Sequencing.}

To subscribe to Genome Research go to: https://genome.cshlp.org/subscriptions 